\title{
Concept Maps
}

Arindam Basu

1 University of Canterbury

Concept maps are graphical representation of knowledge where concepts or patterns are connected to each other using key phrases referred to as linked words. The concepts can be nouns or adjectives, and linked phrases can be verbs or prepositions. The connected concepts are referred to as propositions. Different propositions are connected using arrows or connecting lines referred to as edges. More than one root concept is possible. Concept maps are hierarchically organised with abstract concepts are placed on the top of the map and concrete examples or less abstract concepts are placed to the bottom of the map. See this article by Novak et.al ${ }^{[1]}$ for more information.

\section{References}

\section{1. ${ }^{\wedge}$ https://msu.edu/ /uckie/ctools/}

